\title{
The impact of a multidisciplinary small renal mass clinic on patient treatment decisions
}

Danielle Earis, BSc Hons ${ }^{1}$; Chris Wall, $\mathrm{MD}^{2}$; Nicolette Sinclair, $\mathrm{MD}^{2}$; Trustin Domes, $\mathrm{MD}^{3}$; Kunal Jana, MD ${ }^{3}$

${ }^{1}$ College of Medicine, University of Saskatchewan, Saskatoon, SK, Canada; 2Department of Diagnostic Imaging, Division of Interventional Radiology, University of Saskatchewan, Saskatoon, SK, Canada; ${ }^{3}$ Department of Surgery, Division of Urology, University of Saskatchewan, Saskatoon, SK, Canada

Cite as: Earis D, Wall C, Sincleai N, et al. The impact of a multidisciplinary small renal mass clinic on patient treatment decisions. Can Urol Assoc J 2021 September 24; Epub ahead of print. http://dx.doi.org/10.5489/cuaj.7307

Published online September 24, 2021

Corresponding author: Ms. Danielle Earis, College of Medicine, University of Saskatchewan, Saskatoon, SK, Canada danielle.earis@usask.ca

$$
* * *
$$

\section{Abstract}

Introduction: Small renal masses (SRMs) are managed with active surveillance (AS), thermal ablation (TA), irreversible electroporation (IRE), or surgery, depending on patient and tumor factors. A novel SRM multidisciplinary clinic (SRMC), involving urologists and interventional radiologists, was established to provide patients with information on treatments options. The objective of this study was to evaluate the impact of the SRMC on treatment decision-making Methods: Demographics, tumor characteristics, and treatment decisions were prospectively collected on patients ( $\mathrm{n}=216)$ attending the SRMC between 2016 and 2019. A retrospective historic cohort $(\mathrm{n}=238)$ seen by urologists was used as a control group. Key variables were analyzed and compared. Patient satisfaction $(\mathrm{n}=27)$ was surveyed and responses were summarized and explored.

Results: Mean age, tumor size, and pathology was similar between groups; however, the SRMC cohort had more male patients $(65.7 \%$ vs. $53.8 \%, \mathrm{p}=0.009)$. Chosen treatment modality differed significantly between cohorts $(\mathrm{p}<0.0001)$. Patients in the historic cohort were treated by AS (41.5\%), surgery (37.9\%), TA (11.9\%), watchful waiting (7.9\%), and IRE (0.8\%). SRMC patients were treated by TA (42.2\%), AS (26.7\%), surgery (21.3\%), IRE (7.6\%), and watchful waiting $(2.2 \%)$. Post-hoc analysis revealed statistically significant differences in proportions of 
AS, TA, IRE, and surgery between cohorts. Patients reported high satisfaction with the collaborative approach.

Conclusions: A multidisciplinary approach may have an impact on patient treatment decisionmaking for SRMs. Consultations involving a urologist and an interventional radiologist resulted in more TA and IRE and less AS and surgery. Future studies should evaluate if these findings occur in other centers.

\section{Introduction}

The incidental detection of small renal masses (SRMs) has increased over the years primarily due to the growing use of cross-sectional imaging. SRMs are defined as enhancing renal tumours $4 \mathrm{~cm}$ or less in greatest dimension and these masses are pathologically diverse. ${ }^{1,2}$ Around $80 \%$ of SRMs are renal cell carcinoma (RCC). The majority are low grade and early in stage with slow growth rates. ${ }^{3}$ The pathological grade and metastatic potential of malignant renal tumours increases with greater size and with certain histological variants. ${ }^{4,5}$ Smaller masses are more likely to be benign, with those less than $3 \mathrm{~cm}$ having a negligible risk of metastasis. ${ }^{6}$

The primary goals of managing SRMs are to limit disease-specific mortality and treatment-related morbidity. Given that $20 \%$ of SRMs are benign and those that are malignant have a low metastatic potential, aggressively treating all of these masses is unnecessary and potentially harmful. ${ }^{7}$ Even with advancements in diagnostic imaging, differentiating benign from malignant SRMs preoperatively remains challenging. ${ }^{2}$ Percutaneous renal mass biopsy is reliable with an overall diagnostic accuracy of $92 \%$; however, it is more invasive and its routine use is controversial and reserved for cases where the results will change clinical management. ${ }^{7,8}$ Therefore, decisions regarding treatment of SRMs continue to be made with indeterminate diagnoses.

Surgical approaches, such as radical nephrectomy (RN) and partial nephrectomy (PN), remain the standard of care for localized renal neoplasms greater than $4 \mathrm{~cm} .{ }^{7}$ Although surgery has excellent oncologic outcomes, ${ }^{9}$ extirpation alone has not improved mortality rates for all patients diagnosed with renal cancer, likely due to the morbidity associated with overtreatment. ${ }^{10}$ Therefore, SRM management has evolved to include less invasive approaches. Active surveillance (AS) is increasingly being utilized for select patients. ${ }^{7}$ Ablative techniques have also grown in popularity as nephron-sparing, cost-effective approaches with low morbidity and complication rates. ${ }^{11}$ Irreversible electroporation (IRE) has been demonstrated as comparable to other thermal ablation (TA) therapies, although it requires general anesthesia and is technically more challenging. ${ }^{12}$ A contemporary study ${ }^{13}$ comparing these major treatment options has shownsimilar short-term oncological outcomes. Given the pros and cons of each treatment 
modality, a balanced multidisciplinary discussion of all treatment options is optimal to guide patient care.

A multidisciplinary approach involves the input of multiple specialists that are relevant in ensuring informed and coordinated treatment plans occur for optimal care. ${ }^{14}$ Regarding cancer management, the use of multidisciplinary teams have been shown to extend overall patient survival ${ }^{15}$ as well as improve clinical decision-making ${ }^{16}$ and cancer staging. ${ }^{17}$ In urological malignancies, this approach has been found to impact clinical decisions and improve patient outcomes. ${ }^{18,19}$ A multidisciplinary approach to SRMs is a novel concept and there is only one study published examining the influence it had in management. ${ }^{20}$ Lallas and colleagues reported that this approach provided the opportunity for patients to fully consider multiple treatment modalities. Furthermore, the consultations were often more extensive, facilitating informed patients to choose management plans that best suited their unique circumstances. ${ }^{20}$

A multidisciplinary SRM clinic (SRMC) was developed in Saskatoon, Saskatchewan in October 2016 consisting of urologists and interventional radiologists. This clinic is the only one of its kind in Canada. The goal of this monthly clinic is to present patients with multiple treatment options so they can make informed decisions about their management. The objective of the current study is to evaluate the impact on treatment decision-making by comparing patients having gone through the SRMC with a historical cohort seen only by urologists. The secondary objective is to explore patient satisfaction with the clinic format.

\section{Methods}

\section{Clinic format}

The local health region in Saskatoon, Saskatchewan is a high-volume centre for surgery and ablative techniques for renal masses. Available treatment options at this centre include open and laparoscopic nephrectomy surgery as well as thermal ablation and IRE. The SRMC team includes two urologists and three interventional radiologists (IR). At any given clinic, a combination of one urologist and one IR will participate. Using a pooled referral system, all patients with renal tumours in the local and surrounding area are screened for appropriateness and invited to the clinic when a SRM is confirmed on imaging. During the clinic, the urologist and IR first review the patient's history and radiological findings and then complete the patient consultation together. During the consultation, a history and physical examination are performed and patients are shown their imaging findings. A discussion on utility of a renal mass biopsy and a detailed treatment discussion occurs with both the urologist (leads discussion on AS and surgical options when appropriate) and the IR (leads discussion on TA and IRE when appropriate). If a renal mass biopsy was performed prior to treatment decisions, the SRMC team would have further discussion with the patient after the pathology returned. For patients undergoing TA, renal biopsy was routinely performed prior to treatment when feasible. Final 
treatment decisions were made by the patient through this shared-decision making model after presentation of all information and options.

\section{Study design}

A retrospective chart review of patients referred to Saskatoon Urology Associates between 2012 and 2016 was performed between May and August of 2019. Cases with SRMs were isolated in order to create the historical cohort prior to inception of the SRMC. Exclusion criteria included patients with genetic predispositions for tumours (i.e., von-Hippel Lindau, hereditary papillary $\mathrm{RCC}$ ), radiologically described angiomyolipomas, as well as Bosniak 1,2,2F, and 3 cysts. Collected data were compared to the prospective cohort that had access to the SRMC after its implementation in October 2016. Patients consulted at the clinic during a 2 month period were also asked to complete a survey regarding their experience for quality improvement purposes. Ethics approval for this study was granted by the Biomedical Human Research Ethics Board at the University of Saskatchewan (BIO 1180).

\section{Data collection}

Variables including patient demographics, treatment method, tumour characteristics (diameter, laterality, pathology), and clinical outcome data were extracted from electronic charts at the SRMC and entered into a database. Final pathology results were grouped as RCC, common benign entities (oncocytoma, angiomyolipoma), other non-RCC (e.g., ectopic adrenal adenoma, metanephric adenoma, leiomyoma, metastatic tumour), and unknown (no biopsy performed, nondiagnostic, not amenable, to be determined). Provided treatment modality for each SRM was categorized as AS, TA, IRE, surgery (PN, RN), or watchful waiting when treatment was not beneficial, feasible, or the mass was not the primary malignancy. Average follow-up in months was also calculated using the initial date of consultation and latest available imaging.

Patient satisfaction with the SRMC was collected with a developed survey tool (see Appendix). The survey included a 5-point Likert scale and written response section. Responses ranged from 1 (strongly disagree) to 5 (strongly agree). All patients attending the clinic during a 2 month period were asked to anonymously complete the survey after their visit to evaluate their satisfaction with the clinic model.

\section{Data analysis}

Descriptive analyses for patients' baseline characteristics were performed based on the number of patients in each sample. Univariate analysis was based on each individual SRM and utilized to compare treatment distribution between the historical cohort and prospective SRMC cohort. Categorical comparisons were conducted using Chi-square tests and Fisher's exact test as appropriate. Independent t-tests were used to compare continuous variables between groups. A $p$ value of .05 was considered statistically significant. Post hoc analysis was conducted to assess 
differences of each treatment modality and pathology between cohorts. Chi-square with $p$ values calculated for each cell were compared with a Bonferroni's corrected $p$ value. Corrected $p$ values for treatment modality and pathology categories were .005 and .0062 , respectively. Survey responses were analyzed using central tendency. Themes within written responses were summarised and explored. Statistical analysis was done using SAS 9.4 (SAS Institute, Inc., Cary, NC, USA).

\section{Results}

\section{Demographics and clinical characteristics}

Demographic and clinical characteristics of the historical cohort $(n=238)$ and patients being assessed in the SRMC $(n=216)$ are summarized in Table 1. Given that 21 patients combined in both cohorts had multiple SRMs, a total of 478 SRMs were assigned a treatment decision. No statistically significant difference was found for patient age at time of assessment between the historical and prospective cohorts ( $64.7 \pm 12.5$ vs. $66.0 \pm 11.7$ years). More patients that experienced the SRMC were male $(65.7 \%$ vs. $53.8 \%, p=0.009)$. Tumour size was not statistically significant between the two cohorts $(2.5 \pm 0.8 \mathrm{~cm}$ vs. $2.4 \pm 0.9 \mathrm{~cm})$. The breakdown of SRM pathology is also shown in Table 1 . Final pathology differed significantly between cohorts $(p=0.002)$. However, in post hoc analysis, no categories showed a stastitically significant difference when compared to the Bonferroni's corrected $p$ value of .0062 .

\section{Treatment modality}

Table 1 shows the differences in chosen treatment modality between the pre-SRMC cohort and the post-SRMC cohort. There was a statistically significant difference in the frequency of chosen treatment modality between the cohorts $(p<0.0001)$ : AS $(41.5 \%$ vs. $26.7 \%)$, surgery $(37.9 \%$ vs. $21.3 \%)$, TA ( $11.9 \%$ vs. $42.2 \%)$, IRE (0.8\% vs. $7.6 \%)$ and watchful waiting (7.9\% vs. $2.2 \%)$. Post hoc analysis revealed that between cohorts, the frequency of AS, TA, IRE, and surgery differed significantly from one another. Therefore, after implementation of the SRMC, patients more frequently chose TA and IRE and less frequently chose AS and surgery.

\section{Patient survey}

A survey was utilized to determine patient satisfaction with the SRMC (see Appendix). All patients who participated $(n=27)$ fully completed the survey, with the exception of one respondent who did not indicate an answer for the third question. The mean rating for the questions ranged from 4.65 to 4.89 . Of the responses, the majority of patients strongly agreed with each statement and no patient disagreed with any statements, reporting a high satisfaction rate with the clinic (Figure 1).

Narrative comments revealed themes of increased knowledge transmission and active patient participation in treatment decisions. One participant commented: "It was very beneficial 
to have the team of specialists all together to meet with me and my family to address our concerns. I feel very confident in their diagnosis and treatment and expertise." Another participant noted that the environment contributed to their participation in the conversation about their care: "We appreciate this so much as I like to be knowledgeable about our health situations and be a part of the answers, not the problems." In contrast, one patient mentioned that having multiple doctors at their visit was intimidating and made them "assume the worst."

\section{Discussion}

There are a variety of treatment options for patients presenting with a SRM to consider. The decision on which treatment to pursue can be challenging for patients to make. The influence of this decision by the treatment provider should not be underestimated, with a prior study demonstrating that surgeons are biased towards offering a surgical management approach to treat a SRM. ${ }^{21}$ Similarly, in prostate cancer, physicians are more likely to recommend treatments performed within their specialty. ${ }^{22}$ Multidisciplinary models of care are desirable in these circumstances as they have the potential to improve patient informed decision-making and reduce provider biases.

\section{Multidisciplinary approach}

The objective of this study was to evaluate the impact of a multidisciplinary SRMC, involving urologists and interventional radiologists, on treatment decision-making. The findings suggest that the SRMC may have an impact on patient decision-making regarding treatment for their SRM. Following implementation of the clinic, there was an observed increase in proportions of TA and IRE, which are procedures performed by interventional radiologists. Moreover, there was a significant decrease in patients choosing AS and surgery, which are in the domain of urology. Overall, once consulted by the multidisciplinary team, patients opted for less invasive yet potentially curative approaches over predominantly active surveillance and surgical modalities for their lesions.

The results of this study are consistent with other studies researching the impact of multidisciplinary assessments in urological malignancies. Kurpad and colleagues evaluated the effect of a multidisciplinary tumour board on the diagnosis and treatment decisions of patients ( $n$ =269) newly presenting with a urologic malignancy (prostate, bladder, kidney, testicle, other). The authors found that $38 \%$ of patients experienced a change in their diagnosis and/or treatment, $35 \%$ had no change, $17 \%$ were labelled as "not applicable" (i.e., case was not categorizable to mentioned choices), and $10 \%$ required further analysis. ${ }^{18}$ They found that the impact of these discussions was greatest for lower stage diseases, where more treatment options potentially exist. ${ }^{18}$ Similarly, the impact of collaborative methods have been reported specifically for the treatment of prostate cancer patients. ${ }^{22,23}$ Korman and colleagues reported increased adherence to guidelines and an extension of available treatments for patients who attended the 
multidisciplinary clinic. ${ }^{23}$ In terms of SRM management, only one study has examined the clinical influence of multidisciplinary teams.$^{20}$ Lallas and colleagues assessed associations between treatment choice and several clinical factors at their SRMC. Consistent with our study, the authors concluded that collaborative discussions facilitated informed patient decision-making in one organized visit. ${ }^{20}$ Thus, the multidisciplinary care model appears to facilitate informed decision for SRM patients.

\section{Trends in SRM management}

It is important to consider these results in the context of the evolution of available treatment for SRMs over time. An observed increase in patients choosing ablative therapies (TA, IRE) is consistent with current trends in the contemporary management of SRMs, where the literature has demonstrated long-term oncological efficacy. Over the past several decades, the utilization of ablation has increased when treatment is indicated. ${ }^{1-3,11-13}$ Notably, IRE is a novel treatment option for small solid organ tumours that was not previously available in Saskatchewan centres. This technology is now offered and increasingly used, with preliminary data showing that it is a safe, efficacious option for difficult-to-treat SRMs that would otherwise result in nephrectomy or dialysis. ${ }^{24}$

In a recent study on SRM management trends, Doolittle and colleagues found a $12 \%$ to $30 \%$ rise in ablative therapy throughout 2004 to $2015 .{ }^{25}$ The current study found a similar change in TA, from $12 \%$ to $42 \%$ between 2012 and 2019. Conversely, our results show a general decrease in AS and surgery, a variation that is inconsistent with Doolittle's findings. Doolittle and colleagues reported that rates of AS and surgery were consistent throughout the decade, with an increase in PN and subsequent decrease in $\mathrm{RN} .{ }^{25}$ The variable relation of this study's results to current trends reinforces the specific influence of the SRMC.

\section{Patient satisfaction}

The secondary objective of this study was to assess patient satisfaction with the SRMC using a developed survey. Although several benefits of multidisciplinary approaches have been mentioned above, assumptions must not be made on patient experiences. Therefore, questionnaires for quality improvement efforts are necessary. Survey responses indicated a high level of satisfaction and illustrated benefits of the clinic model. Notably, the clinic provides patients with the opportunity to gather information and discuss available options with the specialists that provide them. Patients also felt encouraged to contribute to the management plan and share their perspective with the team. However, one patient mentioned that it was intimidating to have multiple doctors in the room. Thus, explaining the format and its purpose beforehand may be beneficial. Overall, patients supported the approach, demonstrating the importance of a balanced discussion when implementing a shared decision-making model. These 
findings are similar to previous research that has found high patient satisfaction with multidisciplinary models for cancer management. ${ }^{19,26}$

\section{Study limitations and implications}

This study has several limitations that should be acknowledged. Firstly, the study is limited by its uncontrolled and retrospective design when analysing the historic cohort. As a result, counfounding variables may have influenced treatment decision-making. Secondly, the sample is from a single centre patient population. The historical cohort was seen by 7 to 8 different urological providers, whereas the SRMC comprises of two urologists; thus, provider differences may have affected the results. Furthermore, as different management options may be offered at other centres, the results may not be generalizable across all Canadian provinces. For instance, the observed increase in IRE may be due to growth in availability and funding of the procedure in Saskatchewan. Thirdly, the difference in time between cohorts may have an influence in practice behaviour. Fourthly, this study evaluated the impact of a multidisciplinary SRMC on treatment decision-making without subsequent analysis of clinical outcomes. Although it may be assumed that collaborative approaches lead to informed decisions that improve outcomes, the present study cannot comment on clinical endpoints. Long-term outcomes of the various treatment options have shown to be comparable. ${ }^{13}$ Additionally, the authors have reviewed local, unpublished data on thermal ablation outcomes, which have shown low recurrence and complication rates, and are comparable to established findings in literature. IRE is a novel technique with preliminary data showing that it is an effective option for difficult-to-treat $\mathrm{SRMs}^{24}$; however, long-term outcome data are pending.

In terms of strength, to our knowledge, this is the first study to evaluate the impact of a multidisciplinary SRMC on patient treatment decision-making. The present study extends the understanding of this model for SRMs and sets a foundation for continued research. Future studies should focus on whether clinical and patient-reported outcomes are improved with multidisciplinary models of care for SRMs. Moreover, this study found that less invasive approaches are increasingly utilized over AS and surgery in this setting. The intended and unintended consequences of this change in treatment pattern needs to be evaluated in patients with SRMs to ensure ongoing feasibility, cost, and optimization of oncological and patientreported outcomes. Essentially, the potential of overtreatment by performing more, albeit less invasive, treatments at the expense of decreased AS should be evaluated.

\section{Conclusions}

A multidisciplinary approach has been shown in this single-centre experience to have an impact on treatment decision-making for SRMs. Consultations involving a urologist and an interventional radiologist resulted in patients choosing more procedures performed by interventional radiologists (TA and IRE) and less AS and surgery compared to a historical 
contemporary cohort presenting with a SRM to a urologist only. A multidisciplinary environment enables patients to consider all treatment options in a single setting with the practitioners that perform the various procedures. Patient satisfaction with the SRMC demonstrates the importance of balanced discussions when implementing a shared decisionmaking model. Future studies are required to evaluate if this effect occurs in other centres and to monitor the long-term impact of this change in practice pattern for both the individual patient and the greater healthcare system. 


\section{References}

1. Almassi N, Gill BC, Rini B, et al. Management of the small renal mass. Transl Androl Urol 2017;6:923-30. doi:10.21037/tau.2017.07.11.

2. Sanchez A, Feldman A, Hakimi A. Current management of small renal masses, including patient selection, renal tumor biopsy, active surveillance, and thermal ablation. $J$ Clin Oncol 2018;36:3591-600. doi:10.1200/JCO.2018.79.2341.

3. Gill IS, Aron M, Gervais DA, et al. Clinical practice. Small renal mass. $N$ Engl J Med 2010;362:624-34. doi:10.1056/NEJMcp0910041.

4. Nguyen MM, Gill IS. Effect of renal cancer size on the prevalence of metastasis at diagnosis and mortality. J Urol 2009;181:1020-7. doi:0.1016/j.juro.2008.11.023.

5. Daugherty M, Sedaghatpour D, Shapiro O, et al. The metastatic potential of renal tumors: Influence of histologic subtypes on definition of small renal masses, risk stratification, and future active surveillance protocols. Urol Oncol 2017;35:153.e15-20.

6. Thompson RH, Hill JR, Babayev Y, et al. Metastatic renal cell carcinoma risk according to tumor size. J Urol 2009;182:41-5. doi:10.1016/j.juro.2009.02.128.

7. Jewett MAS, Rendon R, Lacombe L, et al. Canadian guidelines for the management of small renal masses (SRM). Can Urol Assoc J 2015;9:160-3. doi:10.5489/cuaj.2969.

8. Marconi L, Dabestani S, Lam T, et al. Systematic review and meta-analysis of diagnostic accuracy of percutaneous renal tumour biopsy. Eur Urol 2016;69:660-73. doi:10.1016/j.eururo.2015.07.072

9. Sun M, Bianchi M, Trinh QD, et al. Comparison of partial vs radical nephrectomy with regard to other-cause mortality in T1 renal cell carcinoma among patients aged $\geq 75$ years with multiple comorbidities. BJU Int 2013;111:67-73. doi:10.1111/j.1464410X.2012.11254.X

10. Hollingsworth J, Miller D, Daignault S, et al. Rising incidence of small renal masses: A need to reassess treatment effect. J Natl Cancer Inst 2006;98:1331-4. doi:10.1093/jnci/djj362

11. Ha SC, Zlomke HA, Cost N, et al. The past, present, and future in management of small renal masses. J Oncol 2015;2015:364807. doi:10.1155/2015/364807

12. Buijs M, Zondervan P, De Bruin D, et al. Feasibility and safety of irreversible electroporation (IRE) in patients with small renal masses: Results of a prospective study. Urol Oncol 2018;37:183.e1-8. doi:10.1016/j.urolonc.2018.11.008.

13. Alam R, Patel HD, Osumah T, et al. Comparative effectiveness of management options for patients with small renal masses: A prospective cohort study. BJU Int 2019;123:4250. doi:10.1111/bju.14490

14. Fleissig A, Jenkins V, Catt S, et al. Multidisciplinary teams in cancer care: Are they effective in the UK? Lancet Oncol 2006;7:935-43. doi:10.1016/S1470-2045(06)70940-8

15. Junor EJ, Hole DJ, Gillis CR. Management of ovarian cancer: Referral to a multidisciplinary team matters. Br J Cancer 1994;70:363-70. doi:10.1038/bjc.1994.307 
16. Ruhstaller T, Roe H, Thurlimann B, et al. The multidisciplinary meeting: An indispensable aid to communication between different specialities. Eur J Cancer 2006;42:2459-62. doi:10.1016/j.ejca.2006.03.034

17. Stephens MR, Lewis WG, Brewster AE, et al. Multidisciplinary team management is associated with improved outcomes after surgery for esophageal cancer. Dis Esophagus 2006;19:164-71. doi:10.1111/j.1442-2050.2006.00559.x

18. Kurpad R, Kim W, Rathmell WK, et al. A multidisciplinary approach to the management of urologic malignancies: Does it influence diagnostic and treatment decisions? Urol Oncol 2011;29:378-82. doi:10.1016/j.urolonc.2009.04.008

19. Gomella L, Lin J, Hoffman-Censits J, et al. Enhancing prostate cancer care through the multidisciplinary clinic approach: A 15-year experience. J Oncol Pract 2010;6:e5-10. doi:10.1200/JOP.2010.000071

20. Lallas CD, Scotland K, Zhang M, et al. Clinical influences in the multidisciplinary management of small renal masses at a tertiary referral center. Urol Pract 2016;3:468-74. doi:10.1016/j.urpr.2016.02.004

21. Jacobs BL, Tan HJ, Montgomery JS, et al. Understanding criteria for surveillance of patients with a small renal mass. Urol 2012;79:1027-32. doi:10.1016/j.urology.2011.12.052

22. Aizer AA, Paly JJ, Efstathiou JA. Multidisciplinary care and management selection in prostate cancer. Semin Radiat Oncol 2013;23:157-64. doi:10.1016/j.semradonc.2013.01.001

23. Korman H, Lanni T, Shah C, et al. Impact of a prostate multidisciplinary clinic program on patient treatment decisions and on adherence to NCCN guidelines. Am J Clin Oncol 2013;36:121-5. doi:10.1097/COC.0b013e318243708f

24. Liu B, Clark J, Domes T, et al. Percutaneous irreversible electroporation for the treatment of small renal masses: The first Canadian case series. Can Urol Assoc J 2019;13:E263-7. doi:10.5489/cuaj. 5728

25. Doolittle J, Piotrowski J, Zuk K, et al. Evolving trends for selected treatment of T1a renal cell carcinoma. Urol 2019;132:136-42. doi:10.1016/j.urology.2019.06.029

26. Litton G, Kane D, Clay G, et al. Multidisciplinary cancer care with a patient and physician satisfaction focus. J Oncol Pract 2010;6:e35-7. doi:10.1200/JOP.2010.000028 


\section{Figures and Tables}

Fig. 1. Patient survey responses. SRMC: small renal masses multidisciplinary clinic.

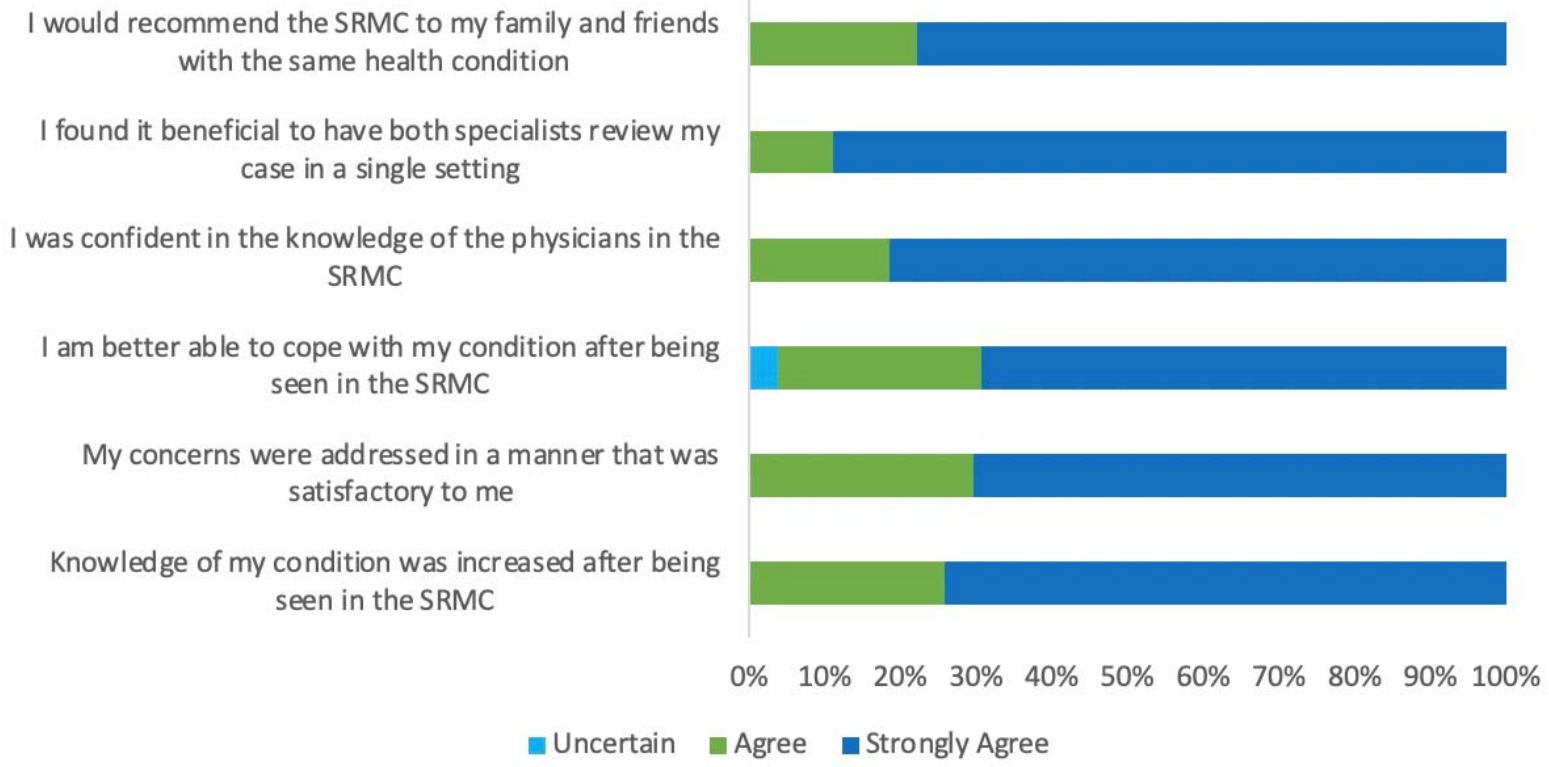
$\begin{array}{llllllllllll}0 \% & 10 \% & 20 \% & 30 \% & 40 \% & 50 \% & 60 \% & 70 \% & 80 \% & 90 \% & 100 \%\end{array}$

- Uncertain agree $\quad$ Strongly Agree

\begin{tabular}{|l|c|c|c|}
\hline \multicolumn{4}{|l|}{ Table 1. Demographic characteristics of patients with SRMs } \\
\hline Characteristic & Historic cohort & SRMC cohort & p \\
\hline Patients & 238 & 216 & \\
\hline Age & $64.7(12.5)$ & $66.0(11.7)$ & 0.25 \\
Mean (SD) & $29-93$ & $32-90$ & \\
Range & & & 0.009 \\
\hline Sex (\%) & $128(53.8)$ & $142(65.7)$ & \\
Male & $110(46.2)$ & $74(34.3)$ & \\
Female & 253 & 225 & \\
\hline SRMs & $105(41.5)$ & $60(26.7)$ & \\
\hline Treatment modality (\%) & $30(11.9)$ & $95(42.2)$ & \\
Active surveillance & $2(0.8)$ & $17(7.6)$ & \\
Thermal ablation & $96(37.9)$ & $48(21.3)$ & \\
Irreversible electroporation & $20(7.9 \%)$ & $5(2.2)$ & \\
Surgery & & & 0.83 \\
Watchful waiting* & & & \\
\hline Size & & & \\
\hline
\end{tabular}




\begin{tabular}{|l|c|c|c|}
\hline Mean (SD) & $2.5(0.8)$ & $2.4(0.9)$ & \\
Range & $0.8-4.0$ & $0.5-4.0$ & \\
\hline Laterality (\%) & $125(49.4)$ & $120(53.3)$ & 0.39 \\
Left & $128(50.6)$ & $105(46.7)$ & \\
Right & & & 0.002 \\
\hline Pathology (\%) & $27(10.7)$ & $32(14.2)$ & \\
Benign & $108(42.7)$ & $122(54.2)$ & \\
RCC & $8(3.2)$ & $1(0.004)$ & \\
Other non-RCC & $110(43.4)$ & $70(31.1)$ & \\
Unknown & & \\
\hline
\end{tabular}

${ }^{*}$ Watchful waiting = treatment was not beneficial, feasible, or the mass was not the primary malignancy. RCC: renal cell carcinoma; SD: standard deviation; SRMC: small renal masses multidisciplinary clinic. 